\title{
New results for fractional differential equations with impulses via variational methods
}

\author{
Peiluan $\mathrm{Li}^{\mathrm{a}, *}$, Hui Wang ${ }^{\mathrm{b}}$, Zheqing $\mathrm{Li}^{\mathrm{c}}$ \\ a School of Mathematics and Statistics, Henan University of Science and Technology, Luoyang, 471023, China. \\ ${ }^{b}$ College of Information Engineering, Henan University of Science and Technology, Luoyang, 471003, China. \\ ${ }^{c}$ Network and Information Center, Henan University of Science and Technology, Luoyang, 471003, China.
}

Communicated by $R$. Saadati

\begin{abstract}
By using variational methods and some critical points theorems, we establish some new results for the existence of infinitely many of solutions for fractional order differential equations with impulses. In addition, one example is given to illustrate our results. (C) 2017 all rights reserved.
\end{abstract}

Keywords: Fractional differential equations, impulses, infinitely many solutions, critical points theorem. 2010 MSC: 26A33, 34B37, 34K10.

\section{Introduction}

Fractional calculus refers to integration or differentiation of any order. The field has a history as old as calculus itself, which did not attract enough attention for a long time. In the past decades, the theory of fractional differential equations has become an important area of investigation because of its wide applicability in many branches of physics, economics and technical sciences. For a nice introduction, we refer the reader to $[6,12-14]$ and references cited therein. The main methods used in the research of fractional differential equations are some fixed theorems, the coincidence degree theory and the monotone iterative methods. On the other hand, critical point theory and the variational methods have been very useful in dealing with the existence and multiplicity of solutions for integer order differential equations with some boundary conditions. We refer readers to the books (or surveys) and the papers [5, 16, 19] and the references therein. The variational methods are also a very useful tool in dealing with the fractional differential equations; see [1, 4, 10, 11, 15, 22-24]. In [10], Jiao and Zhou considered the following fractional boundary value problems

$$
\left\{\begin{array}{l}
-\frac{1}{2} \frac{d}{d t}\left({ }_{0} D_{t}^{-\beta}+{ }_{t} D_{T}^{-\beta}\right) u^{\prime}(t)=\nabla F(t, u(t)), \\
u(0)=u(T)=0
\end{array}\right.
$$

\footnotetext{
*Corresponding author

Email addresses: lpllpl_lpl@163.com (Peiluan Li), wh@haust.edu.cn (Hui Wang), lzq@haust.edu.cn (Zheqing Li)
} 
where $\beta \in[0,1),{ }_{0} D_{t}^{-\beta}$ and ${ }_{t} D_{T}^{-\beta}$ are the left and right Riemann-Loiuville fractional derivatives, respectively. $F:[0, T] \times R^{N} \rightarrow R$ (with $N \geqslant 1$ ) is a suitable given function and $\nabla F(t, x)$ is the gradient of $F$ with respect to $x$.

By using the least action principle and Mountain Pass theorem, they obtained some sufficient conditions for the existence of one solution. The authors in $[4,8,9,15,22]$ further studied the existence and multiplicity of solutions for the above problem or related problems with the critical point theory. And the above problem arises from the phenomena of advection dispersion and was first investigated by Ervin and Loop in [7].

Impulsive effects are common phenomena due to short-term perturbations whose duration is negligible in comparison with the total duration of the original process. The governing equations of such phenomena may be modeled as impulsive differential equations. In recent years, there has been a growing interest in the study of impulsive differential equations as these equations provide a natural framework for mathematical modeling of many real world phenomena, namely in the control theory, physics, chemistry, population dynamics, biotechnology, economics, and medical fields [17, 21].

Recently, the study of fractional differential equations with impulses has been studied by many authors. But for almost all the works, the main methods are some fixed theorems, the coincidence degree theory, and the monotone iterative methods. To our best knowledge, the fractional boundary value problems with impulses using variational methods and critical point theory have received considerably less attention [3, 18, 20]. Bonanno et al. [3] and Rodríguez-López and Tersian [20] studied the following Dirichlet's boundary value problem for fractional differential equations with impulses:

$$
\begin{cases}\left.{ }_{t} D_{T}^{\alpha}{ }_{0}^{c} D_{t}^{\alpha} u(t)\right)+a(t) u(t)=\lambda f(t, u(t)), & t \neq t_{j}, \text { a.e. } t \in[0, T], \\ \triangle\left({ }_{t} D_{T}^{\alpha-1}\left({ }_{0}^{c} D_{t}^{\alpha} u\right)\right)\left(t_{j}\right)=\mu I_{j}\left(u\left(t_{j}\right)\right), & j=1,2, \ldots, n, \\ u(0)=u(T)=0, & \end{cases}
$$

where $\lambda \in(0,+\infty)$ and $\mu \in(0,+\infty)$ are two parameters. They obtained the existence of triple solutions by using variatioanl methods and a three critical points theorem due to Bonanno and Marano [2].

In [18], the authors investigated the following fractional differential equations with impulses:

$$
\begin{cases}{ }_{t} D_{T}^{\alpha}\left({ }_{0}^{c} D_{t}^{\alpha} u(t)\right)+a(t) u(t)=f(t, u(t)), & t \neq t_{j}, \text { a.e. } t \in[0, T], \\ \triangle\left({ }_{t} D_{T}^{\alpha-1}\left({ }_{0}^{c} D_{t}^{\alpha} u\right)\right)\left(t_{j}\right)=I_{j}\left(u\left(t_{j}\right)\right), & j=1,2, \ldots, n, \\ u(0)=u(T)=0 . & \end{cases}
$$

By using critical point theory and variational methods, the authors give some criteria to guarantee that the above-mentioned impulsive problems have at least one solution and infinitely many solutions.

Motivated by the work above, we consider the following problem (1.1) of impulsive fractional differential equations:

$$
\begin{cases}-\frac{1}{2} \frac{d}{d t}\left({ }_{0} D_{t}^{-\beta}+{ }_{t} D_{T}^{-\beta}\right) u^{\prime}(t)=a(t) u(t)+\lambda \nabla F(t, u(t)), & t \neq t_{k}, \text { a.e. } t \in[0, T], \\ \triangle\left(D_{t}^{\alpha} u\right)\left(t_{k}\right)=\mu I_{k}\left(u\left(t_{k}\right)\right), & t_{k} \in(0, T), k=1,2, \ldots, l, \\ u(0)=u(T)=0, & \end{cases}
$$

where $\beta \in[0,1), \alpha=1-\frac{\beta}{2} \in\left(\frac{1}{2}, 1\right],{ }_{0} D_{t}^{-\beta},{ }_{t} D_{T}^{-\beta}$ are the left and right Riemann-Liouville fractional integrals of order $\beta,{ }_{0}^{c} D_{t}^{\alpha}$ and ${ }_{t}^{c} D_{T}^{\alpha}$ are the left and right Caputo fractional derivative of order $\alpha$, respectively, $0=t_{0}<t_{1}<t_{2}<\ldots<t_{l}<t_{l+1}=T, F:[0, T] \times R^{N} \rightarrow R$ a given function satisfying some assumptions and $\nabla F(t, x)$ is the gradient of $F$ at $x, I_{k} \in C([0, T], R), a \in C[0, T]$ and

$$
\begin{aligned}
\left(D_{t}^{\alpha} u\right)(t) & =\frac{1}{2}\left\{{ }_{0} D_{t}^{\alpha-1}\left({ }_{0}^{c} D_{t}^{\alpha} u\right)-{ }_{t} D_{T}^{\alpha-1}\left({ }_{t}^{c} D_{T}^{\alpha} u\right)\right\}(t), \\
\triangle\left(D_{t}^{\alpha} u\right)\left(t_{k}\right) & =\frac{1}{2}\left\{{ }_{0} D_{t}^{\alpha-1}\left({ }_{0}^{c} D_{t}^{\alpha} u\right)-{ }_{t} D_{T}^{\alpha-1}\left({ }_{t}^{c} D_{T}^{\alpha} u\right)\right\}\left(t_{k}^{+}\right)
\end{aligned}
$$




$$
\begin{aligned}
& -\frac{1}{2}\left\{{ }_{0} D_{t}^{\alpha-1}\left({ }_{0}^{c} D_{t}^{\alpha} \mathfrak{u}\right)-{ }_{t} D_{T}^{\alpha-1}\left({ }_{t}^{c} D_{T}^{\alpha} u\right)\right\}\left(t_{k}^{-}\right), \\
& \frac{1}{2}\left\{{ }_{0} D_{t}^{\alpha-1}\left({ }_{0}^{c} D_{t}^{\alpha} u\right)-{ }_{t} D_{T}^{\alpha-1}\left({ }_{t}^{c} D_{T}^{\alpha} u\right)\right\}\left(t_{k}^{+}\right)=\lim _{t \rightarrow t_{k}^{+}} \frac{1}{2}\left\{{ }_{0} D_{t}^{\alpha-1}\left({ }_{0}^{c} D_{t}^{\alpha} u\right)-{ }_{t} D_{T}^{\alpha-1}\left({ }_{t}^{c} D_{T}^{\alpha} u\right)\right\}(t), \\
& \frac{1}{2}\left\{{ }_{0} D_{t}^{\alpha-1}\left({ }_{0}^{c} D_{t}^{\alpha} u\right)-{ }_{t} D_{T}^{\alpha-1}\left({ }_{t}^{c} D_{T}^{\alpha} u\right)\right\}\left(t_{k}^{-}\right)=\lim _{t \rightarrow t_{k}^{-}} \frac{1}{2}\left\{{ }_{0} D_{t}^{\alpha-1}\left({ }_{0}^{c} D_{t}^{\alpha} \mathfrak{u}\right)-{ }_{t} D_{T}^{\alpha-1}\left({ }_{t}^{c} D_{T}^{\alpha} u\right)\right\}(t)
\end{aligned}
$$

for $k=1, \cdots, l$.

In this paper, the existence results of infinitely many solutions of (1.1) are established. Our method is different from $[3,18,20]$ and our results for (1.1) are new. The rest of this paper is organized as follows. In Section 2, some definitions and lemmas which are essential to prove our main results are stated. In Section 3, we give the main results. At last, one example is offered to demonstrate the application of our main results.

\section{Preliminaries}

At first, we present the necessary definitions for the fractional calculus theory and several lemmas which are used further in this paper.

Definition 2.1 ([12]). Let $f$ be a function defined on $[a, b]$. The left and right Riemann-Liouville fractional integrals of order $\alpha$ for function $f$ are denoted by ${ }_{a} D_{t}^{-\alpha}$ and ${ }_{t} D_{b}^{-\alpha}$, respectively, and are defined by

$$
\begin{aligned}
& { }_{a} D_{t}^{-\alpha} f(t)=\frac{1}{\Gamma(\alpha)} \int_{a}^{t}(t-s)^{\alpha-1} f(s) d s, \\
& { }_{t} D_{b}^{-\alpha} f(t)=\frac{1}{\Gamma(\alpha)} \int_{t}^{b}(s-t)^{\alpha-1} f(s) d s,
\end{aligned}
$$

for $t \in[a, b], \alpha>0$.

The left and right Riemann-Liouville fractional derivatives of order $\alpha$ for function $f$ are denoted by ${ }_{a} D_{t}^{-\alpha} f(t)$ and ${ }_{t} D_{b}^{-\alpha} f(t)$, respectively, and are defined by

$$
\begin{aligned}
& { }_{a} D_{t}^{-\alpha} f(t)=\frac{d^{n}}{d t^{n}}{ }_{a} D_{t}^{\alpha-n} f(t)=\frac{1}{\Gamma(n-\alpha)} \frac{d^{n}}{d t^{n}} \int_{a}^{t}(t-s)^{n-\alpha-1} f(s) d s, \\
& { }_{t} D_{b}^{-\alpha} f(t)=(-1)^{n} \frac{d^{n}}{d t^{n}}{ }_{t} D_{b}^{\alpha-n} f(t)=\frac{(-1)^{n}}{\Gamma(n-\alpha)} \frac{d^{n}}{d t^{n}} \int_{t}^{b}(s-t)^{n-\alpha-1} f(s) d s,
\end{aligned}
$$

for $\mathrm{t} \in[\mathrm{a}, \mathrm{b}], \mathrm{n}-1 \leqslant \alpha<\mathrm{n}, \mathrm{n} \in \mathrm{N}$.

The above conditions ensure that the right hand side integral is well-defined as pointwise on $[a, b]$.

Lemma 2.2 ([12]). The left and right Riemann-Liouville fractional integral operators have the property of a semigroup, i.e.

$$
{ }_{a} D_{t}^{-\gamma_{1}}\left({ }_{a} D_{t}^{-\gamma_{2}} f(t)\right)={ }_{a} D_{t}^{-\gamma_{1}-\gamma_{2}} f(t) \text { and }{ }_{t} D_{b}^{-\gamma_{1}}\left({ }_{t} D_{b}^{-\gamma_{2}} f(t)\right)={ }_{t} D_{b}^{-\gamma_{1}-\gamma_{2}} f(t), \forall \gamma_{1}, \gamma_{2}>0,
$$

for a.e. $\mathrm{t} \in[\mathrm{a}, \mathrm{b}]$ and $\mathrm{f} \in \mathrm{L}^{1}\left([\mathrm{a}, \mathrm{b}], \mathrm{R}^{\mathrm{N}}\right)$.

Definition 2.3 ([12]). If $\alpha \in(n-1, n)$ and $f \in A C^{n}([a, b], R)$, then the left and right Caputo fractional derivatives of order of a function $f$ are denoted by ${ }_{a}^{c} D_{t}^{\alpha} f(t)$ and ${ }_{t}^{c} D_{b}^{\alpha} f(t)$, respectively, and are defined by

$$
\begin{aligned}
& { }_{a}^{c} D_{t}^{\alpha} f(t)={ }_{a} D_{t}^{\alpha-n} \frac{d^{n}}{d t^{n}} f(t)=\frac{1}{\Gamma(n-\alpha)} \int_{a}^{t}(t-s)^{n-\alpha-1} f^{(n)}(s) d s, \\
& { }_{t}^{c} D_{b}^{\alpha} f(t)=(-1)^{n}{ }_{t} D_{b}^{\alpha-n} \frac{d^{n}}{d t^{n}} f(t)=\frac{(-1)^{n}}{\Gamma(n-\alpha)} \int_{t}^{b}(s-t)^{n-\alpha-1} f^{(n)}(s) d s,
\end{aligned}
$$

for $t \in[a, b]$. 
In view of Definition 2.1 and Lemma 2.2, we can easily transfer (1.1) to the following problem

$$
\left\{\begin{array}{rlrl}
\frac{d}{d t}\left\{\frac{1}{2}{ }_{0} D_{t}^{\alpha-1}\left({ }_{0}^{c} D_{t}^{\alpha} u(t)\right)-\frac{1}{2}{ }_{t} D_{T}^{\alpha-1}\left({ }_{t}^{c} D_{T}^{\alpha} u(t)\right)\right\} & & t \neq t_{k}, \text { a.e. } t \in[0, T], \\
& \quad+a(t) u(t)+\lambda \nabla F(t, u(t))=0, & \\
\triangle\left(D_{t}^{\alpha} \mathfrak{u}\right)\left(t_{k}\right)=\mu I_{k}\left(u\left(t_{k}\right)\right), & t_{k} \in(0, T), k=1,2, \ldots, l, \\
u(0)=u(T)=0, &
\end{array}\right.
$$

where $\alpha=1-\frac{\beta}{2} \in\left(\frac{1}{2}, 1\right],{ }_{0} D_{t}^{\alpha-1},{ }_{t} D_{T}^{\alpha-1}$ are the left and right Riemann-Liouville fractional integrals of order $1-\alpha,{ }_{0}^{c} D_{t}^{\alpha}$ and ${ }_{t}^{c} D_{T}^{\alpha}$ are the left and right Caputo fractional derivative of order $\alpha$, respectively, and $\left(D_{t}^{\alpha} \mathfrak{u}\right)\left(t_{k}\right)=\frac{1}{2}\left\{{ }_{0} D_{t}^{\alpha-1}\left({ }_{0}^{c} D_{t}^{\alpha} \mathfrak{u}\right)-{ }_{t} D_{T}^{\alpha-1}\left({ }_{t}^{c} D_{T}^{\alpha} u\right)\right\}\left(t_{k}\right)$ for $k=1, \ldots, l$.

Then the problem (1.1) is equivalent to the problem (2.1). Therefore a solution of the problem (2.1) corresponds to a solution of the BVP (1.1).

In order to establish a variational structure which enables us to reduce the existence of solution of problem (2.1) to the existence of the critical point of corresponding functional, we construct the following appropriate function spaces.

Let us recall that for any fixed $t \in[0, T]$ and $1 \leqslant p \leqslant \infty$,

$$
\|u\|_{\infty}=\max _{t \in[0, T]}|u(t)|, \quad\|u\|_{L^{p}}=\left(\int_{0}^{T}|u(s)|^{p} d s\right)^{\frac{1}{p}} .
$$

Let $0<\alpha \leqslant 1$, we define the fractional derivative spaces $E_{0}^{\alpha}$ by the closure of $C_{0}^{\infty}([0, T])$ with respect to the weighted norm $\|u\|_{\alpha}=\left(\int_{0}^{T}\left|{ }_{0}^{c} D_{t}^{\alpha} u(t)\right|^{2} d t+\int_{0}^{T}|u(t)|^{2} d t\right)^{\frac{1}{2}}$, for all $u \in E_{0}^{\alpha}$, where

$$
\mathrm{C}_{0}^{\infty}([0, \mathrm{~T}])=\left\{u \in \mathrm{C}_{0}^{\infty}([0, \mathrm{~T}]): \mathrm{u}(0)=\mathrm{u}(\mathrm{T})\right\} .
$$

Clearly, the fractional derivative space $E_{0}^{\alpha}$ is the space of functions $u \in L^{2}\left([0, T] \backslash\left\{t_{1}, t_{2}, \ldots, t_{l}\right\}\right)$ having $\alpha$-order Caputo left and right fractional derivatives and Riemann-Liouville left and right fractional derivatives, ${ }_{0}^{c} \mathrm{D}_{\mathrm{t}}^{\alpha},{ }_{\mathrm{t}}^{c} \mathrm{D}_{\mathrm{T}}^{\alpha},{ }_{0} \mathrm{D}_{\mathrm{t}}^{\alpha} \in \mathrm{L}^{2}\left([0, \mathrm{~T}] \backslash\left\{\mathrm{t}_{1}, \mathrm{t}_{2}, \ldots, \mathrm{t}_{\mathrm{l}}\right\}\right)$ and $\mathrm{u}(0)=\mathrm{u}(\mathrm{T})=0$.

Lemma 2.4 ([10]). Let $\frac{1}{2}<\alpha \leqslant 1$ and $1<\mathrm{p}<\infty$ for all $\mathrm{u} \in \mathrm{E}_{0}^{\alpha}$, one has

$$
\|u\|_{L^{p}} \leqslant \frac{T^{\alpha}}{\Gamma(\alpha+1)}\left\|{ }_{0}^{c} D_{t}^{\alpha} u\right\|_{L^{p}} .
$$

Moreover, if $\alpha>\frac{1}{p}$ and $\frac{1}{p}+\frac{1}{q}=1$, then

$$
\|u\|_{\infty} \leqslant \frac{T \alpha-1 / p}{\Gamma(\alpha)((\alpha-1) q+1)^{\frac{1}{q}}}\left\|{ }_{0}^{c} D_{t}^{\alpha} u\right\|_{L^{p}} .
$$

Then we can conclude that $\|\mathrm{u}\|_{\alpha}=\left(\int_{0}^{\mathrm{T}}\left|{ }_{0}^{\mathrm{c}} \mathrm{D}_{\mathrm{t}}^{\alpha} \mathfrak{u}(\mathrm{t})\right|^{2} \mathrm{~d} \mathrm{t}+\int_{0}^{\mathrm{T}}|\mathrm{u}(\mathrm{t})|^{2} \mathrm{dt}\right)^{\frac{1}{2}}$ is equivalent to $\|\mathrm{u}\|_{\alpha}=\left(\int_{0}^{\mathrm{T}}\left|{ }_{0}^{\mathrm{c}} \mathrm{D}_{\mathrm{t}}^{\alpha} \mathrm{u}(\mathrm{t})\right|^{2} \mathrm{dt}\right)^{\frac{1}{2}}$, for all $\mathrm{u} \in \mathrm{E}_{0}^{\alpha}$.

In the following, we will consider the fractional derivative spaces $E_{0}^{\alpha}$ with respect to the norm $\|u\|_{\alpha}=$ $\left(\int_{0}^{T}\left|{ }_{0}^{c} D_{t}^{\alpha} u(t)\right|^{2} d t\right)^{\frac{1}{2}}$. By the discussion in [10], we know $E_{0}^{\alpha}$ is a reflexive and separable Banach space with the norm $\|u\|_{\alpha}=\left(\int_{0}^{T}\left|{ }_{0}^{c} D_{t}^{\alpha} u(t)\right|^{2} d t\right)^{\frac{1}{2}}$.

The following concepts are similar to Definition 2.1 and 2.2 in [3].

Definition 2.5.

$$
u \in\left\{u \in A C([0, T]): \int_{t_{j}}^{t_{j+1}}\left(\int_{0}^{T}\left|{ }_{0}^{c} D_{t}^{\alpha} u(t)\right|^{2}+\int_{0}^{T}|u(t)|^{2}\right)^{\frac{1}{2}} d t<\infty, j=0, \ldots, l\right\},
$$

is called a classic solution of the problem (2.1) if 
(i)

$$
\begin{aligned}
& \frac{1}{2}\left\{{ }_{0} D_{t}^{\alpha-1}\left({ }_{0}^{c} D_{t}^{\alpha} u\right)-{ }_{t} D_{T}^{\alpha-1}\left({ }_{t}^{c} D_{T}^{\alpha} u\right)\right\}\left(t_{k}^{+}\right)=\lim _{t \rightarrow t_{k}^{+}} \frac{1}{2}\left\{{ }_{0} D_{t}^{\alpha-1}\left({ }_{0}^{c} D_{t}^{\alpha} u\right)-{ }_{t} D_{T}^{\alpha-1}\left({ }_{t}^{c} D_{T}^{\alpha} u\right)\right\}(t), \\
& \frac{1}{2}\left\{{ }_{0} D_{t}^{\alpha-1}\left({ }_{0}^{c} D_{t}^{\alpha} u\right)-{ }_{t} D_{T}^{\alpha-1}\left({ }_{t}^{c} D_{T}^{\alpha} u\right)\right\}\left(t_{k}^{-}\right)=\lim _{t \rightarrow t_{k}^{-}} \frac{1}{2}\left\{{ }_{0} D_{t}^{\alpha-1}\left({ }_{0}^{c} D_{t}^{\alpha} u\right)-{ }_{t} D_{T}^{\alpha-1}\left({ }_{t}^{c} D_{T}^{\alpha} u\right)\right\}(t),
\end{aligned}
$$

exist and satisfy the impulsive condition $\triangle\left(D_{t}^{\alpha}\right)\left(t_{k}\right)=I_{k}\left(u\left(t_{k}\right)\right)$ and the boundary condition $u(0)=$ $\mathrm{u}(\mathrm{T})=0$ holds;

(ii) $u$ satisfies (1.1) a.e. on $t \in[0, T] \backslash\left\{t_{1}, t_{2}, \ldots, t_{l}\right\}$.

Definition 2.6. A function $u \in E_{0}^{\alpha}$ is called a weak solution of the problem (2.1) if

$$
\begin{aligned}
\int_{0}^{T}\{ & \left.-\frac{1}{2}\left[{ }_{0}^{c} D_{t}^{\alpha} u(t){ }_{t}^{c} D_{T}^{\alpha} v(t)+{ }_{t}^{c} D_{T}^{\alpha} u(t){ }_{0}^{c} D_{t}^{\alpha} v(t)\right]-a(t) u(t) v(t)\right\} d t \\
& +\mu \sum_{j=1}^{l} I_{j}\left(u\left(t_{j}\right)\right) v\left(t_{j}\right)-\lambda \int_{0}^{T} \nabla F(t, u(t)) v(t) d t=0,
\end{aligned}
$$

for all $v(\mathrm{t}) \in \mathrm{E}_{0}^{\alpha}$.

Similar to the proof of Lemma 2.1 in [3], we have the following lemma.

Lemma 2.7. The function $\mathrm{u} \in \mathrm{E}_{0}^{\alpha}$ is a classical solution of (2.1) if and only if $\mathrm{u}$ is a weak solution of (2.1).

Proof. By standard arguments, if $u \in \mathrm{E}_{0}^{\alpha}$ is a classical solution of (2.1), then $u$ is a weak solution of (2.1). Conversely, let $u \in \mathrm{E}_{0}^{\alpha}$ be a weak solution of (2.1), we prove that $u$ is a classical solution of (2.1).

If $u \in E_{0}^{\alpha}$ is a weak solution of (2.1), for a fixed $j \in\{0,1, \ldots, l\}$, we choose a function $w_{j}$ such that $w_{j}=0$, for $t \in\left[0, t_{j}\right] \cup\left[t_{j+1}, T\right]$. Similar to the proof of Lemma 2.1 in [3], we can obtain

$$
\int_{t_{j}}^{t_{j+1}}-\frac{1}{2}\left[{ }_{0}^{c} D_{t}^{\alpha} u(t){ }_{t}^{c} D_{T}^{\alpha} w_{j}(t)+{ }_{t}^{c} D_{T}^{\alpha} u(t){ }_{0}^{c} D_{t}^{\alpha} w_{j}(t)\right] d t=\int_{t_{j}}^{t_{j+1}}[a(t) u(t)+\lambda \nabla F(t, u(t))] w_{j}(t) d t
$$

with

$$
\int_{t_{j}}^{t_{j+1}}-\frac{1}{2}\left[{ }_{0}^{c} D_{t}^{\alpha} u(t){ }_{t}^{c} D_{T}^{\alpha} w_{j}(t)+{ }_{t}^{c} D_{T}^{\alpha} u(t){ }_{0}^{c} D_{t}^{\alpha} w_{j}(t)\right] d t<\infty,
$$

which shows

$$
\frac{d}{d t}\left\{\frac{1}{2}{ }_{0} D_{t}^{\alpha-1}\left({ }_{0}^{c} D_{t}^{\alpha} u(t)\right)-\frac{1}{2}{ }_{t} D_{T}^{\alpha-1}\left({ }_{t}^{c} D_{T}^{\alpha} u(t)\right)\right\}+a(t) u(t)+\lambda \nabla F(t, u(t))=0,
$$

for almost all $t \in\left(t_{j}, t_{j+1}\right)$. Then $u$ satisfies the first equation of (2.1) for almost all $t \in(0, T)$.

Also similar to the proof of Lemma 2.1 in [3], we can prove

$$
\frac{1}{2}\left\{{ }_{0} D_{t}^{\alpha-1}\left({ }_{0}^{c} D_{t}^{\alpha} u\right)-{ }_{t} D_{T}^{\alpha-1}\left({ }_{t}^{c} D_{T}^{\alpha} u\right)\right\} \in A C\left(\left[t_{j}, t_{j+1}\right]\right),
$$

and there exist the limits

$$
\begin{aligned}
& \frac{1}{2}\left\{{ }_{0} D_{t}^{\alpha-1}\left({ }_{0}^{c} D_{t}^{\alpha} u\right)-{ }_{t} D_{T}^{\alpha-1}\left({ }_{t}^{c} D_{T}^{\alpha} u\right)\right\}\left(t_{k}^{+}\right)=\lim _{t \rightarrow t_{k}^{+}} \frac{1}{2}\left\{{ }_{0} D_{t}^{\alpha-1}\left({ }_{0}^{c} D_{t}^{\alpha} u\right)-{ }_{t} D_{T}^{\alpha-1}\left({ }_{t}^{c} D_{T}^{\alpha} u\right)\right\}(t), \\
& \frac{1}{2}\left\{{ }_{0} D_{t}^{\alpha-1}\left({ }_{0}^{c} D_{t}^{\alpha} u\right)-{ }_{t} D_{T}^{\alpha-1}\left({ }_{t}^{c} D_{T}^{\alpha} u\right)\right\}\left(t_{k}^{-}\right)=\lim _{t \rightarrow t_{k}^{-}} \frac{1}{2}\left\{{ }_{0} D_{t}^{\alpha-1}\left({ }_{0}^{c} D_{t}^{\alpha} u\right)-{ }_{t} D_{T}^{\alpha-1}\left({ }_{t}^{c} D_{T}^{\alpha} u\right)\right\}(t) .
\end{aligned}
$$


Multiplying $\triangle\left(D_{t}^{\alpha} u\right)\left(t_{k}\right)$ by $v \in E_{0}^{\alpha}$ and integrating by parts, due to the definition of weak solution and Proposition 2.4 in [3], we can get

$$
\sum_{k=1}^{l} \triangle\left(D_{t}^{\alpha} \mathfrak{u}\right)\left(t_{k}\right) v\left(t_{k}\right)=\sum_{k=1}^{l} \mu I_{k}\left(u\left(t_{k}\right)\right) v\left(t_{k}\right) .
$$

Let $v_{j}\left(t_{k}\right)=\delta_{j k}$, it follows from (2.4) that $\triangle\left(D_{t}^{\alpha} u\right)\left(t_{k}\right)=\mu I_{k}\left(u\left(t_{k}\right)\right), k=1,2, \ldots, l$. Then the impulsive conditions of (2.1) are satisfied. Hence $u$ is a classical solution of (2.1).

Similar to some properties in [10], we have the following results.

Lemma 2.8. Let $0<\alpha \leqslant 1$. The fractional derivative space $\mathrm{E}_{0}^{\alpha}$ is a reflexive and a separable Banach space.

Lemma 2.9. Let $\frac{1}{2}<\alpha \leqslant 1$ and the sequence $\left\{\mathfrak{u}_{k}\right\}$ converges weakly to $u$ in $\mathrm{E}_{0}^{\alpha}$. Then $\mathfrak{u}_{k} \rightarrow \mathfrak{u}$ in $\mathrm{C}([0, \mathrm{~T}], \mathrm{R})$, that is, $\left\|\mathrm{u}_{\mathrm{k}}-\mathrm{u}\right\|_{\infty} \rightarrow 0$ as $\mathrm{k} \rightarrow \infty$.

Lemma 2.10. Let $\frac{1}{2}<\alpha \leqslant 1$. For any $\mathrm{u} \in \mathrm{E}_{0}^{\alpha}$, one has

$$
|\cos (\pi \alpha)|\|u\|_{\alpha}^{2} \leqslant-\int_{0}^{T}{ }_{0}^{c} D_{t}^{\alpha} u(t){ }_{t}^{c} D_{T}^{\alpha} u(t) d t \leqslant \frac{1}{|\cos (\pi \alpha)|}\|u\|_{\alpha}^{2} .
$$

Proof. Noticing that $\cos (\pi \alpha) \in[-1,0)$ for $\alpha \in\left(\frac{1}{2}, 1\right]$, by calculation, we have

$$
-\int_{0}^{T}{ }_{0}^{c} D_{t}^{\alpha} u(t){ }_{t}^{c} D_{T}^{\alpha} u(t) d t \geqslant-\cos (\pi \alpha) \int_{0}^{T}\left|{ }_{0} D_{t}^{\alpha} u(t)\right|^{2} d t=|\cos (\pi \alpha)| \int_{0}^{T}\left|{ }_{0}^{c} D_{t}^{\alpha} u(t)\right|^{2} d t=|\cos (\pi \alpha)|\|u\|_{\alpha}^{2} .
$$

By using Young's inequality and a standard derivation, we can get another inequality.

For more details, one can see Proposition 4.1 in [10].

Finally, we recall the following critical point theorem.

Lemma 2.11. Let $X$ be a reflexive real Banach space $\Phi, \Psi: X \rightarrow R$ be two Gâteaux differentiable functionals such that $\Phi$ is sequentially weakly lower semi-continuous, strongly continuous, and coercive, and $\Psi$ is sequentially weakly upper semicontinuous. For every $\mathrm{r}>\inf _{\mathrm{X}} \Phi$, let us put

$$
\varphi(r)=\inf _{u \in \Phi^{-1}(]-\infty, r[)} \frac{\sup _{v \in \Phi^{-1}(]-\infty, r[)} \Psi(v)-\Psi(u)}{r-\Phi(u)}
$$

and

$$
\gamma=\liminf _{r \rightarrow+\infty} \varphi(r), \quad \delta=\liminf _{r \rightarrow\left(\inf _{X} \Phi\right)^{+}} \varphi(r) .
$$

(1) If $\gamma<+\infty$, then, for each $\lambda \in] 0, \frac{1}{\gamma}$ [, the following alternative holds: either the functional $\Phi-\lambda \Psi$ has a global minimum, or there exists a sequence $\left\{u_{n}\right\}$ of critical points (local minima) of $\Phi-\lambda \Psi$ such that $\lim _{n \rightarrow+\infty} \Phi\left(u_{n}\right)=$ $+\infty$.

(2) If $\delta<+\infty$, then, for each $\lambda \in] 0, \frac{1}{\delta}$ [, the following alternative holds: either there exists a global minimum of $\Phi$ which is a local minimum of $\Phi-\lambda \Psi$, or there exists a sequence $\left\{u_{n}\right\}$ of pairwise distinct critical points (local minima) of $\Phi-\lambda \Psi$, with $\lim _{n \rightarrow+\infty} \Phi\left(u_{n}\right)=\inf _{X} \Phi$, which weakly converges to a global minimum of $\Phi$.

\section{Main results}

Define

$$
\Phi(u)=\int_{0}^{T}-\frac{1}{2}{ }_{0}^{c} D_{t}^{\alpha} u(t){ }_{t}^{c} D_{T}^{\alpha} u(t) d t-\frac{1}{2} \int_{0}^{T} a(t) u^{2}(t) d t,
$$




$$
\Psi(u)=\int_{0}^{T} F(t, u(t)) d t-\frac{\mu}{\lambda} \sum_{j=1}^{l} \int_{0}^{u\left(t_{j}\right)} I_{j}(s) d s .
$$

Clearly, $\Phi, \Psi$ are Gâteaux differentiable functionals whose Gâteaux derivatives at the point $u \in \mathrm{E}_{0}^{\alpha}$ are given by

$$
\begin{aligned}
& \Phi^{\prime}(\mathrm{u}) v=\int_{0}^{T}-\frac{1}{2}\left[{ }_{0}^{\mathrm{c}} \mathrm{D}_{\mathrm{t}}^{\alpha} \mathrm{u}(\mathrm{t}){ }_{\mathrm{t}}^{\mathrm{c}} \mathrm{D}_{\mathrm{T}}^{\alpha} v(\mathrm{t})+{ }_{\mathrm{t}}^{\mathrm{c}} \mathrm{D}_{\mathrm{T}}^{\alpha} \mathrm{u}(\mathrm{t}){ }_{0}^{\mathrm{c}} \mathrm{D}_{\mathrm{t}}^{\alpha} v(\mathrm{t})\right] \mathrm{dt}-\int_{0}^{\mathrm{T}} \mathrm{a}(\mathrm{t}) \mathrm{u}(\mathrm{t}) v(\mathrm{t}) \mathrm{dt}, \\
& \Psi^{\prime}(\mathrm{u}) v=\int_{0}^{T} \nabla \mathrm{F}(\mathrm{t}, \mathrm{u}(\mathrm{t})) v(\mathrm{t}) \mathrm{dt}-\frac{\mu}{\lambda} \sum_{j=1}^{\mathrm{l}} \mathrm{I}_{j}\left(\mathrm{u}\left(\mathrm{t}_{\mathrm{j}}\right)\right) v\left(\mathrm{t}_{\mathrm{j}}\right) .
\end{aligned}
$$

Then the critical point of $\Phi-\lambda \Psi$ is exactly the weak solution of the problem (2.1). By Lemma 2.7, it is the classical solution of the problem (2.1). By the equivalence of (1.1) and (2.1), we know it is also a classical solution of (1.1).

Throughout this paper, we assume that

$\left(\mathrm{H}_{1}\right) \lambda, \mu>0$ are real parameters, $\beta \in[0,1), \alpha=1-\frac{\beta}{2} \in\left(\frac{1}{2}, 1\right], \mathrm{F}:[0, \mathrm{~T}] \times \mathrm{R} \rightarrow \mathrm{R}$ is a function such that $F(\cdot, x)$ is continuous in $[0, T]$ for every $x \in R, F(\cdot, x)$ is a $C^{1}$-function in $R$ for any $t \in[0, T]$, and $\nabla F(t, x)$ is the gradient of $F$ at $x, a \in C[0, T]$ and there exist two positive constants $a_{1}$ and $a_{2}$ such that $0<a_{1} \leqslant a \leqslant a_{2}, I_{j} \in C\left([0, T], R^{+}\right), j=1, \ldots, l$.

By the assumptions $\left(\mathrm{H}_{1}\right),(3.1),(3.2)$, we can easily get the following lemma.

Lemma 3.1. Let $\alpha \in\left(\frac{1}{2}, 1\right]$ and assumption $\left(\mathrm{H}_{1}\right)$ be satisfied. If $\mathrm{u} \in \mathrm{E}_{0}^{\alpha}$, then the functional $\Phi: \mathrm{E}^{\alpha} \rightarrow \mathrm{R}^{\mathrm{N}}$ denoted by (3.1) is convex and continuous on $\mathrm{E}_{0}^{\alpha}$.

Proof. From Definition 2.3, $\left(\mathrm{H}_{1}\right)$, and (3.1), it is easy to see that $\Phi: E^{\alpha} \rightarrow R^{N}$ is continuous. From $\left(H_{1}\right)$, by a standard argument, we can easily prove that $\frac{1}{2} \int_{0}^{T} a(t) u^{2}(t) d t$ is convex. So in order to show $\Phi$ is convex, we only need to prove the convexity of $\int_{0}^{T}-\frac{1}{2}{ }_{0}^{c} D_{t}^{\alpha} u(t){ }_{t}^{c} D_{T}^{\alpha} u(t) d t$.

We denote

$$
\mathrm{H}(\mathrm{u})=\int_{0}^{T}-\frac{1}{2}{ }_{0}^{\mathrm{c}} \mathrm{D}_{\mathrm{t}}^{\alpha} \mathrm{u}(\mathrm{t}){ }_{\mathrm{t}}^{\mathrm{c}} \mathrm{D}_{\mathrm{T}}^{\alpha} \mathrm{u}(\mathrm{t}) \mathrm{dt} .
$$

Let $\lambda \in(0,1), u, v \in E_{0}^{\alpha}, \bar{u}, \bar{v}$ be the extension of $u$ and $v$ by zero on $\mathrm{R} /[0, \mathrm{~T}]$. Since the Caputo fractional derivative operator is linear operator, then by Lemma 5.1 in [10], we have

$$
\begin{aligned}
H[(1-\lambda) u+\lambda v] & =-\frac{1}{2} \int_{0}^{T}{ }_{0}^{c} D_{t}^{\alpha}[(1-\lambda) u(t)+\lambda v(t)]{ }_{t}^{c} D_{T}^{\alpha}[(1-\lambda) u(t)+\lambda v(t)] d t \\
& =\frac{|\cos (\pi \alpha)|}{2} \int_{-\infty}^{+\infty}\left|{ }_{-\infty} D_{t}^{\alpha}[(1-\lambda) \bar{u}(t)+\lambda \bar{v}(t)]\right|^{2} d t \\
& \leqslant \frac{|\cos (\pi \alpha)|}{2} \int_{-\infty}^{+\infty}\left[\left.\left.(1-\lambda)\right|_{-\infty} D_{t}^{\alpha} \bar{u}(t)\right|^{2}+\lambda\left|{ }_{-\infty} D_{t}^{\alpha} \bar{v}(t)\right|^{2}\right] d t \\
& =\int_{0}^{T}\left[-\frac{1-\lambda}{2}\left({ }_{0}^{c} D_{t}^{\alpha} u(t){ }_{t}^{c} D_{T}^{\alpha} u(t)\right)-\frac{\lambda}{2}\left({ }_{0}^{c} D_{t}^{\alpha} v(t){ }_{t}^{c} D_{T}^{\alpha} v(t)\right)\right] d t \\
& =(1-\lambda) H(u)+\lambda H(v) .
\end{aligned}
$$

Then $H(u)$ is convex on $E_{0}^{\alpha}$, which also implies the convexity of $\Phi$. The proof is complete.

For convenience, we put

$$
M=\Gamma(\alpha) \sqrt{\frac{(2 \alpha-1)}{\mathrm{T}\left[|\cos (\pi \alpha)| \mathrm{T}^{2(\alpha-1)}-\mathrm{a}_{2} \Gamma^{2}(\alpha)(2 \alpha-1)\right]}},
$$




$$
\lambda_{1}=\frac{1}{2\left[M^{2} \mathrm{~T}(\mathrm{~d}+1)+\mathrm{B}(\alpha, \mathrm{T})\right]}, \quad \lambda_{2}=\frac{1}{2 \mathrm{M}^{2} \mathrm{~T}(\mathrm{~d}+1)},
$$

where

$$
\begin{aligned}
B(\alpha, T)= & \frac{36}{T^{2}}\left\{\frac{1+\left(\frac{1}{6}\right)^{3-2 \alpha}+\left(\frac{5}{6}\right)^{3-2 \alpha}}{3-2 \alpha} T^{3-2 \alpha}-2 \int_{\frac{T}{6}}^{T}\left[t\left(t-\frac{1}{6} T\right)\right]^{(1-\alpha)} d t\right. \\
& \left.-2 \int_{\frac{5 T}{6}}^{T}\left[\left(t\left(t-\frac{5 T}{6}\right)\right)^{(1-\alpha)}-\left(\left(t-\frac{1}{6} T\right)\left(t-\frac{5 T}{6}\right)\right)^{(1-\alpha)}\right] d t\right\} .
\end{aligned}
$$

Theorem 3.2. Let $\left(\mathrm{H}_{1}\right)$ hold. Assume

$$
\frac{7 \mathrm{~B}(\alpha, \mathrm{T})}{3|\cos (\pi \alpha)| \Gamma^{2}(2-\alpha) \mathrm{T}}<\mathrm{a}_{1} \leqslant \mathrm{a}_{2}<\frac{|\cos (\pi \alpha)|}{\Gamma^{2}(\alpha)(2 \alpha-1)} \mathrm{T}^{2(\alpha-1)} .
$$

If there exists a positive constant $\mu_{0}$ such that $\mu_{0} \mathrm{~b} \leqslant \frac{\mathrm{a}_{1}}{8}$ and the following hypotheses $\left(\mathrm{H}_{2}\right)-\left(\mathrm{H}_{3}\right)$ are satisfied:

$\left(\mathrm{H}_{2}\right) \mathrm{F}(\mathrm{t}, \mathrm{u}) \geqslant 0$ for $(\mathrm{t}, \mathrm{u}) \in[0, \mathrm{~T}] \times \mathrm{R}^{+}$and $\limsup _{\mathrm{s} \rightarrow+\infty} \frac{\mathrm{F}(\mathrm{t}, \mathrm{s})}{\mathrm{s}^{2}}=\mathrm{d}<+\infty$, for a.e $\mathrm{t} \in[0, \mathrm{~T}]$.

$\left(\mathrm{H}_{3}\right)$ For any $\mathrm{j}=1, \ldots, \mathrm{l}$, there exists $b_{j}>0$ such that

$$
\limsup _{|s| \rightarrow \infty} \frac{I_{j}(s)}{s^{2}} \leqslant b_{j}
$$

where $b=\max _{1 \leqslant j \leqslant l} b_{j}$. Then for any $\left.\lambda \in\right] \lambda_{1}, \lambda_{2}\left[, \mu \in\left[0, \mu_{0}[\right.\right.$, the problem (1.1) possesses an unbounded sequence of solutions in $\mathrm{E}_{0}^{\alpha}$.

Proof. We note that $\left(\mathrm{H}_{3}\right)$ implies there exist a positive constant $\mathrm{a}_{3}$ large enough such that

$$
I_{j}(s)<b_{j} s^{2} \text { for }|s|>a_{3}, j=1, \ldots, l .
$$

For any $j=1, \ldots, l$, from the continuity of $I_{j}(s)$, we conclude that $I_{j}(s)$ is bounded for $|s| \leqslant a_{3}, t \in[0, T]$. Then there exist constants $c_{j}>0$ such that

$$
\mathrm{I}_{\mathrm{j}}(\mathrm{s})<\mathrm{c}_{\mathrm{j}},
$$

for $|s| \leqslant a_{3}, j=1, \ldots, l$.

Hence, for $s \in R, j=1, \ldots, l$, we can get

$$
\mathrm{I}_{j}(\mathrm{~s})<\mathrm{c}_{j}+\mathrm{b}_{j} \mathrm{~s}^{2} .
$$

Then it follows that

$$
\sum_{j=1}^{\mathrm{l}} \int_{0}^{\mathfrak{u}\left(\mathrm{t}_{j}\right)} \mathrm{I}_{j}(s) d s \leqslant b \int_{0}^{\mathrm{T}} \mathrm{s}^{2} \mathrm{~d} s+\mathrm{c}_{0} \mathrm{~T},
$$

where $b=\max _{1 \leqslant j \leqslant l} b_{j}, c_{0}=\max _{1 \leqslant j \leqslant l} c_{j}$.

From $\left(\mathrm{H}_{2}\right)$, there exists a positive constant $e_{0}$ large enough such that

$$
F(t, s)<(d+1) s^{2} \text { for }|s|>e_{0} .
$$

From $\left(H_{1}\right)$, we know $F(t, \cdot)$ is a function in $R$ for any $t \in[0, T]$. So we conclude that $F(t, s)$ is bounded for $|s| \leqslant e_{0}, t \in[0, T]$. Then there exists a constant $d_{1}>0$ such that

$$
|\mathrm{F}(\mathrm{t}, \mathrm{s})|<\mathrm{d}_{1},
$$


for $|s| \leqslant e_{0}, t \in[0, T]$.

Hence, for $s \in R, t \in[0, T]$, we have

$$
|F(t, s)|<d_{1}+(d+1) s^{2} .
$$

Now, we prove all the conditions of Lemma 2.11 hold.

By Lemma 3.1 and $\left(\mathrm{H}_{1}\right)$, we know that $\Phi$ is continuously Gâteaux differentiable and convex. Let $u_{n} \rightarrow \mathfrak{u}$ weakly in $E_{0}^{\alpha}$, by Lemma 2.9 , it is easy to know

$$
\liminf _{n \rightarrow \infty} \Phi\left(u_{n}\right)=\liminf _{n \rightarrow \infty} \int_{0}^{T}-\frac{1}{2}{ }_{0}^{c} D_{t}^{\alpha} u_{n}(t){ }_{t}^{c} D_{T}^{\alpha} u_{n}(t) d t \geqslant \int_{0}^{T}-\frac{1}{2}{ }_{0}^{c} D_{t}^{\alpha} u(t){ }_{t}^{c} D_{T}^{\alpha} u(t) d t=\Phi(u),
$$

so $\Phi$ is weakly sequentially lower semi-continuous.

Moreover, it follows from (2.2) and Lemma 2.10 that $\Phi$ is coercive.

From $\left(\mathrm{H}_{1}\right)$ and (3.2), we can conclude that the functional $\Psi$ is continuously Gâteaux differentiable with $\Psi^{\prime}(\mathfrak{u}) v=\int_{0}^{T} \nabla \mathrm{F}(\mathrm{t}, \mathrm{u}(\mathrm{t})) v(\mathrm{t}) \mathrm{dt}-\frac{\mathrm{u}}{\lambda} \sum_{j=1}^{l} \mathrm{I}_{j}\left(u\left(\mathrm{t}_{\mathfrak{j}}\right)\right) v\left(\mathrm{t}_{\mathfrak{j}}\right)$. By a standard procedure (see [19]), we can prove the derivative of $\Psi$ is compact. By Fatou's Lemma, one has

$$
\limsup _{n \rightarrow \infty} \int_{0}^{T} F\left(t, u_{n}(t)\right) d t \leqslant \int_{0}^{T} F(t, u(t)) d t .
$$

Then we can easily get that $\Psi$ is sequentially weakly upper semicontinuous.

Next, we divide our proof into three steps.

Step 1. We prove that $\gamma<+\infty$. Let $\rho_{n}$ be a sequence of positive numbers such that $\lim _{n \rightarrow \infty} \rho_{n}=+\infty$. We choose $r_{n}=\frac{\rho_{n}^{2}}{2 M^{2}}$ for all $n \in N$. For $v \in E_{0}^{\alpha}$, if $\Phi(v) \leqslant r_{n}$, by Lemma 2.10 and (2.3), it follows that

$$
\begin{aligned}
\frac{\rho_{n}^{2}}{2 M^{2}}=r_{n} \geqslant \Phi(v) & =\int_{0}^{T}-\frac{1}{2}{ }_{0}^{c} D_{t}^{\alpha} u(t){ }_{t}^{c} D_{T}^{\alpha} u(t) d t-\frac{1}{2} \int_{0}^{T} a(t) u^{2}(t) d t \\
& >\frac{1}{2}\left[|\cos (\pi \alpha)|\|u\|_{\alpha}^{2}-a_{2} T\|u\|_{\infty}^{2}\right] \\
& >\frac{1}{2}\left[|\cos (\pi \alpha)| \frac{T^{2 \alpha-1}}{\Gamma^{2}(\alpha)(2 \alpha-1)}-a_{2} T\right]\|u\|_{\infty}^{2} .
\end{aligned}
$$

By (3.3), we know

$$
\frac{1}{2}\left[|\cos (\pi \alpha)| \frac{\mathrm{T}^{2 \alpha-1}}{\Gamma^{2}(\alpha)(2 \alpha-1)}-\mathrm{a}_{2} \mathrm{~T}\right]>0 .
$$

Then it is easily gotten that $\|u\|_{\infty} \leqslant \rho_{n}$, which implies that

$$
\Phi^{-1}(]-\infty, r_{n}[)=\left\{v: \Phi(v)<r_{n}\right\} \subseteq\left\{v:\|v\|_{\infty}<\rho_{n}\right\} \subseteq\left\{v:|v|<\rho_{n}\right\} .
$$

It follows from (3.2), (3.5), and $\left(\mathrm{H}_{1}\right)$ that

$$
\begin{aligned}
\gamma=\liminf _{r \rightarrow+\infty} \varphi(r) \leqslant \liminf _{n \rightarrow+\infty} \varphi\left(r_{n}\right) & =\liminf _{n \rightarrow+\infty} \inf _{u \in \Phi^{-1}(]-\infty, r_{n}[)} \frac{\sup _{v \in \Phi^{-1}(]-\infty, r_{n}[)} \Psi(v)-\Psi(u)}{\sup _{n}-\Phi(u)} \Psi(v) \\
& \leqslant \liminf _{n \rightarrow+\infty} \frac{v \in \Phi^{-1}(]-\infty, r_{n}[)}{r_{n}} \\
& \leqslant \lim _{n \rightarrow \infty} \sup _{|v|<\rho_{n}} 2 M^{2} \frac{\int_{0}^{T} F(t, v(t)) d t}{\rho_{n}^{2}} \\
& \leqslant \lim _{n \rightarrow \infty} 2 M^{2} \frac{T\left[d_{1}+(d+1) \rho_{n}^{2}\right]}{\rho_{n}^{2}} \\
& =2 M^{2} T(d+1)<+\infty
\end{aligned}
$$


Step 2. We show that $] \lambda_{1}, \lambda_{2}[\subset] 0, \frac{1}{\gamma}[$.

From the proof of Step 1, we have

$$
\gamma \geqslant 2 M^{2} T(d+1)=\frac{1}{\lambda_{2}}
$$

Assume $\left\{x_{n}\right\}$ is a positive real sequence with $x_{n} \rightarrow+\infty$ as $n \rightarrow+\infty$. We define functions $\omega_{n}$ by

$$
\omega_{n}= \begin{cases}\frac{6 \Gamma(2-\alpha) x_{n} t}{T}, & t \in\left[0, \frac{T}{6}\right), \\ \Gamma(2-\alpha) x_{n}, & t \in\left[\frac{T}{6}, \frac{5 T}{6}\right], \\ \frac{6 \Gamma(2-\alpha) x_{n}}{T}(T-t), & t \in\left(\frac{5 T}{6}, T\right] .\end{cases}
$$

It is easy to verify that $\omega_{n}(0)=\omega_{n}(T)=0$ and $\omega_{n} \in L^{2}[0, T]$. By Definition 2.3, we can directly calculate the left Caputo fractional derivative of order $\alpha$ for $\omega_{n}$.

If $t \in\left[0, \frac{T}{6}\right)$, one has

$$
{ }_{0}^{C} D_{t}^{\alpha} f(t)=\frac{1}{T(1-\alpha)} \int_{0}^{t}(t-s)^{-\alpha} \frac{6 \Gamma(2-\alpha) x_{n}}{T} d s=\left.\frac{6 \Gamma(2-\alpha) x_{n}}{T} \frac{(t-s)^{1-\alpha}}{1-\alpha}\right|_{t} ^{0}=\frac{6 x_{n} t^{1-\alpha}}{T} .
$$

If $t \in\left[\frac{T}{6}, \frac{5 T}{6}\right]$, we get

$$
\begin{aligned}
{ }_{0}^{C} D_{t}^{\alpha} f(t) & =\frac{1}{T(1-\alpha)} \int_{0}^{t}(t-s)^{-\alpha} f^{\prime}(s) d s \\
& =\frac{1}{T(1-\alpha)}\left[\int_{0}^{\frac{T}{6}}(t-s)^{-\alpha} \frac{6 \Gamma(2-\alpha) x_{n}}{T} d s+\int_{\frac{T}{6}}^{t}(t-s)^{-\alpha} 0 d s\right] \\
& =\left.\frac{6 \Gamma(2-\alpha) x_{n}}{T} \frac{(t-s)^{1-\alpha}}{1-\alpha}\right|_{\frac{T}{6}} ^{0}=\frac{6 x_{n}\left(t^{1-\alpha}-\left(t-\frac{T}{6}\right)^{1-\alpha}\right)}{T} .
\end{aligned}
$$

If $t \in\left(\frac{5 T}{6}, T\right]$, we can obtain

$$
\begin{aligned}
{ }_{0}^{C} D_{t}^{\alpha} f(t) & =\frac{1}{T(1-\alpha)} \int_{0}^{t}(t-s)^{-\alpha} f^{\prime}(s) d s \\
& =\frac{1}{T(1-\alpha)}\left[\int_{0}^{\frac{T}{6}}(t-s)^{-\alpha} \frac{6 \Gamma(2-\alpha) x_{n}}{T} d s+\int_{\frac{T}{6}}^{\frac{5 T}{6}}(t-s)^{-\alpha} 0 d s-\int_{\frac{5 T}{6}}^{t}(t-s)^{-\alpha} \frac{6 \Gamma(2-\alpha) x_{n}}{T} d s\right] \\
& =\frac{6 \Gamma(2-\alpha) x_{n}}{T}\left[\left.\frac{(t-s)^{1-\alpha}}{1-\alpha}\right|_{\frac{T}{6}} ^{0}-\left.\frac{(t-s)^{1-\alpha}}{1-\alpha}\right|_{t} ^{\frac{5 T}{6}}\right] \\
& =\frac{\left.6 x_{n}\left[t^{1-\alpha}-\left(t-\frac{T}{6}\right)^{1-\alpha}\right)-\left(t-\frac{5 T}{6}\right)^{1-\alpha}\right]}{T} .
\end{aligned}
$$

Then

$$
\begin{aligned}
\left\|\omega_{n}\right\|_{\alpha}^{2} & =\int_{0}^{T}\left[{ }_{0}^{C} D_{t}^{\alpha} \omega_{n}(t)\right]^{2} d t \\
& =\int_{0}^{\frac{T}{6}}\left(\frac{6 x_{n} t^{1-\alpha}}{T}\right)^{2} d t+\int_{\frac{T}{6}}^{\frac{5 T}{6}}\left[\frac{6 x_{n}\left(t^{1-\alpha}-\left(t-\frac{T}{6}\right)^{1-\alpha}\right)}{T}\right]^{2} d t
\end{aligned}
$$




$$
\begin{aligned}
& +\int_{\frac{5 T}{6}}^{T}\left[\frac{6 x_{n}\left[t^{1-\alpha}-\left(t-\frac{T}{6}\right)^{1-\alpha}-\left(t-\frac{5 T}{6}\right)^{1-\alpha}\right]}{T}\right]^{2} d t \\
= & \frac{36 x_{n}^{2}}{T^{2}}\left\{\int_{0}^{T} t^{2(1-\alpha)} d t+\int_{\frac{T}{6}}^{\frac{5 T}{6}}\left(t-\frac{1}{6} T\right)^{2(1-\alpha)} d t+\int_{\frac{5 T}{6}}^{T}\left(t-\frac{5 T}{6}\right)^{2(1-\alpha)} d t\right. \\
& \left.-2 \int_{\frac{T}{6}}^{T}\left[t\left(t-\frac{1}{6} T\right)\right]^{(1-\alpha)} d t-2 \int_{\frac{5 T}{6}}^{T}\left[\left(t\left(t-\frac{5 T}{6}\right)\right)^{(1-\alpha)}-\left(\left(t-\frac{T}{6}\right)\left(t-\frac{5 T}{6}\right)\right)^{(1-\alpha)}\right] d t\right\} \\
= & \frac{36 x_{n}^{2}}{T^{2}}\left\{\frac{1+\left(\frac{1}{6}\right)^{3-2 \alpha}+\left(\frac{5}{6}\right)^{3-2 \alpha}}{3-2 \alpha} T^{3-2 \alpha}-2 \int_{\frac{T}{6}}^{T}\left[t\left(t-\frac{1}{6} T\right)\right]^{(1-\alpha)} d t\right. \\
& \left.-2 \int_{\frac{5 T}{6}}^{T}\left[\left(t\left(t-\frac{5 T}{6}\right)\right)^{(1-\alpha)}-\left(\left(t-\frac{T}{6}\right)\left(t-\frac{5 T}{6}\right)\right)^{(1-\alpha)}\right] d t\right\} \\
= & B(\alpha, T) x_{n}^{2} .
\end{aligned}
$$

So we can see $B(\alpha, T)=\frac{\left\|\omega_{n}\right\|_{\alpha}^{2}}{x_{n}^{2}} \geqslant 0$. It follows that

$$
0<\lambda_{1}=\frac{1}{2\left[\mathrm{M}^{2} \mathrm{~T}(\mathrm{~d}+1)+\mathrm{B}(\alpha, \mathrm{T})\right]}<\lambda_{2}
$$

Together with (3.6) and (3.7), we can obtain that

$$
] \lambda_{1}, \lambda_{2}[\subset] 0, \frac{1}{\gamma}[.
$$

Step 3. We verify that the operator $\Phi-\lambda \Psi$ is unbounded from below, which implies $\Phi-\lambda \Psi$ has no global minimum. By (3.1) and Lemma 2.10, for $\omega_{n} \in E_{0}^{\alpha}$, one has

$$
\begin{aligned}
\Phi\left(\omega_{n}\right) & =\int_{0}^{T}-\frac{1}{2}{ }_{0}^{c} D_{t}^{\alpha} \omega_{n}(t){ }_{t}^{c} D_{T}^{\alpha} \omega_{n}(t) d t-\frac{1}{2} \int_{0}^{T} a(t) \omega_{n}^{2}(t) d t \\
& \leqslant \frac{1}{2|\cos (\pi \alpha)|}\left\|\omega_{n}\right\|_{\alpha}^{2}-\frac{1}{2} \int_{0}^{T} a(t) \omega_{n}^{2}(t) d t \\
& =\frac{1}{2}\left[\frac{1}{|\cos (\pi \alpha)|} B(\alpha, T) x_{n}^{2}-\int_{0}^{T} a(t) \omega_{n}^{2}(t) d t\right] .
\end{aligned}
$$

From (3.2) and (3.4), for $\omega_{n} \in E_{0}^{\alpha}$, and any $\left.\lambda \in\right] \lambda_{1}, \lambda_{2}\left[, \mu \in\left[0, \mu_{0}[\right.\right.$, we have

$$
\begin{aligned}
\Psi(\Psi) & =\int_{0}^{T} F\left(t, \omega_{n}(t)\right) d t-\frac{\mu}{\lambda} \sum_{j=1}^{l} \int_{0}^{u\left(t_{j}\right)} I_{j}(s) d s \\
& >\int_{0}^{T} F\left(t, \omega_{n}(t)\right) d t-\frac{\mu_{0}}{\lambda}\left(b \int_{0}^{T} \omega_{n}^{2} d s+c_{0} T\right) \\
& =\int_{0}^{T} F\left(t, \omega_{n}(t)\right) d t-\frac{\mu_{0} b}{\lambda} \int_{0}^{T} \omega_{n}^{2}(t) d t-\frac{c_{0} \mu_{0} T}{\lambda} .
\end{aligned}
$$

It follows from (3.3), (3.8), (3.9), and the condition $\mu_{0} b \leqslant \frac{a_{1}}{8}$ that

$$
\begin{aligned}
\Phi\left(\omega_{n}\right)-\lambda \Psi\left(\omega_{n}\right)< & \frac{1}{2}\left[\frac{1}{|\cos (\pi \alpha)|} B(\alpha, T) x_{n}^{2}-\frac{1}{2} \int_{0}^{T} a(t) \omega_{n}^{2}(t) d t .\right] \\
& -\lambda \int_{0}^{T} F\left(t, \omega_{n}(t)\right) d t+\mu_{0} b \int_{0}^{T} \omega_{n}^{2}(t) d t+c_{0} \mu_{0} T
\end{aligned}
$$




$$
\begin{aligned}
\leqslant & \frac{1}{2}\left[\frac{1}{|\cos (\pi \alpha)|} B(\alpha, T) x_{n}^{2}-\int_{0}^{T} a(t) \omega_{n}^{2}(t) d t+\mu_{0} b \int_{0}^{T} \omega_{n}^{2}(t) d t+c_{0} \mu_{0} T\right. \\
= & \frac{1}{2|\cos (\pi \alpha)|} B(\alpha, T) x_{n}^{2}-\left(\frac{a_{1}}{2}-\mu_{0} b\right)\left\{\int_{0}^{\frac{T}{6}}\left[\frac{6 \Gamma(2-\alpha) x_{n} t}{T}\right]^{2} d t\right. \\
& \left.+\int_{\frac{T}{6}}^{\frac{5 T}{6}}\left[\Gamma(2-\alpha) x_{n}\right]^{2} d t+\int_{\frac{5 T}{6}}^{T}\left[\frac{6 \Gamma(2-\alpha) x_{n}}{T}(T-t)\right]^{2} d t\right\}+c_{0} \mu_{0} T \\
< & \frac{1}{2|\cos (\pi \alpha)|} B(\alpha, T) x_{n}^{2}-\left(\frac{a_{1}}{2}-\mu_{0} b\right) \int_{\frac{T}{6}}^{\frac{5 T}{6}}\left[\Gamma(2-\alpha) x_{n}\right]^{2} d t+c_{0} \mu_{0} T \\
= & x_{n}^{2}\left[\frac{B(\alpha, T)}{2|\cos (\pi \alpha)|}-\frac{2 T \Gamma^{2}(2-\alpha)}{3}\left(\frac{a_{1}}{2}-\mu_{0} b\right)\right]+c_{0} \mu_{0} T \\
< & -\frac{B(\alpha, T)}{12|\cos (\pi \alpha)|} x_{n}^{2}+c_{0} \mu_{0} T \\
\rightarrow & -\infty, \text { as } n \rightarrow+\infty .
\end{aligned}
$$

Hence, one can obtain

$$
\lim _{n \rightarrow+\infty} \Phi\left(\omega_{n}\right)-\lambda \Psi\left(\omega_{n}\right)=-\infty,
$$

which yields $\Phi-\lambda \Psi$ is unbounded from below, then $\Phi-\lambda \Psi$ has no global minimum.

So all the conditions of case 1 in Lemma 2.11 are satisfied. By virtue of Lemma 2.11, the functional $\Phi-\lambda \Psi$ admits a sequence $\left\{u_{n}\right\}$ of critical points (local minima) such that $\lim _{n \rightarrow+\infty} \Phi\left(u_{n}\right)=+\infty$. It follows from (3.1) and Lemma 2.10 that

$$
\begin{aligned}
\Phi\left(u_{n}\right) & =\int_{0}^{T}-\frac{1}{2}{ }_{0}^{c} D_{t}^{\alpha} u_{n}(t){ }_{t}^{c} D_{T}^{\alpha} u_{n}(t) d t-\frac{1}{2} \int_{0}^{T} a(t) u_{n}^{2}(t) d t \\
& \leqslant \int_{0}^{T}-\frac{1}{2}{ }_{0}^{c} D_{t}^{\alpha} u_{n}(t){ }_{t}^{c} D_{T}^{\alpha} u_{n}(t) d t \leqslant \frac{1}{2|\cos (\pi \alpha)|}\|u\|_{\alpha^{\prime}}^{2}
\end{aligned}
$$

which implies $\lim _{n \rightarrow+\infty}\left\|u_{n}\right\|_{\alpha}^{2}=+\infty$. Then $\left\{u_{n}\right\}$ is an unbounded sequence. That is, the boundary value problem (2.1) has an unbounded sequence of weak solutions. As a consequence of Lemma 2.7, we deduce that the boundary value problem (2.1) possesses an unbounded sequence of classical solutions in $\mathrm{E}_{0}^{\alpha}$. Then the problem (1.1) has an unbounded sequence of classical solutions in $E_{0}^{\alpha}$.

Remark 3.3. The assumption $\left(\mathrm{H}_{2}\right)$ includes the asymptotically quadratic case and subquadratic case for $F(t, s)$. If $F(t, s)$ in $\left(H_{2}\right)$ is superquadratic, we cannot obtain the result by Lemma 2.11. In fact, in this case,

$$
\liminf _{n \rightarrow+\infty} \frac{\sup _{v \in \Phi^{-1}(]-\infty, r_{n}[)} \Psi(v)}{r_{n}} \rightarrow+\infty .
$$

Next, by the case 2 of Lemma 2.11 and a similar proof, we can get the following existence result of infinitely many solutions of (1.1) converging at zero.

We denote

$$
\lambda_{3}=\frac{1}{2\left[M^{2} \mathrm{~T}\left(\mathrm{~d}^{\prime}+1\right)+\mathrm{B}(\alpha, \mathrm{T})\right]}, \quad \lambda_{4}=\frac{1}{2 \mathrm{M}^{2} \mathrm{~T}\left(\mathrm{~d}^{\prime}+1\right)} .
$$

Theorem 3.4. Let $\left(\mathrm{H}_{1}\right)$ hold. Assume

$$
\frac{7 B(\alpha, T)}{3|\cos (\pi \alpha)| \Gamma^{2}(2-\alpha) \mathrm{T}}<\mathrm{a}_{1} \leqslant \mathrm{a}_{2}<\frac{|\cos (\pi \alpha)|}{\Gamma^{2}(\alpha)(2-\alpha)} \mathrm{T}^{2(\alpha-1)} .
$$

If there exists a positive constant $\mu_{0}^{\prime}$ such that $\mu_{0}^{\prime} \mathrm{b}^{\prime}<\frac{\mathrm{a}_{1}}{8}$ and the following hypotheses are satisfied: 
$\left(\mathrm{H}_{2}\right)^{\prime} \mathrm{F}(\mathrm{t}, \mathrm{u}) \geqslant 0$ for $(\mathrm{t}, \mathrm{u}) \in[0, \mathrm{~T}] \times \mathrm{R}^{+}$and $\limsup _{\mathrm{s} \rightarrow 0^{+}} \frac{\mathrm{F}(\mathrm{t}, \mathrm{s})}{\mathrm{s}^{2}}=\mathrm{d}^{\prime}>0$ for a.e. $\mathrm{t} \in[0, \mathrm{~T}]$;

$\left(\mathrm{H}_{3}\right)^{\prime}$ for any $\mathrm{j}=1, \ldots, \mathrm{l}$, there exists $\mathrm{b}_{\mathrm{j}}^{\prime}>0$ such that

$$
\limsup _{|s| \rightarrow 0^{+}} \frac{I_{j}(s)}{s^{2}} \leqslant b_{j}^{\prime}
$$

where $b^{\prime}=\max _{1 \leqslant j \leqslant l} b_{j}^{\prime}$, then for any $\left.\lambda \in\right] \lambda_{3}, \lambda_{4}\left[, \mu \in\left[0, \mu_{0}^{\prime}\left[\right.\right.\right.$, the problem (1.1) possesses a sequence $\left\{u_{n}\right\}$ of solutions with $\left\{\mathrm{u}_{\mathrm{n}}\right\}$ converging strongly to 0 in $\mathrm{E}_{0}^{\alpha}$.

Finally, we give an example to illustrate the usefulness of our main result. Consider the following impulsive system of fractional differential equations.

\section{Example 3.5.}

$$
\left\{\begin{array}{l}
-\frac{1}{2} \frac{d}{d t}\left({ }_{0} D_{t}^{-0.5}+{ }_{t} D_{1}^{-0.5}\right) u^{\prime}(t)=0.069 u(t)+\lambda \nabla F(t, u(t)), t \neq 50, \text { a.e., } t \in[0,100], \\
\triangle\left(D_{t}^{\alpha} u\right)(50)=\mu \frac{u^{2}(50)}{50} \\
u(0)=u(100)=0 .
\end{array}\right.
$$

From (3.10), we can see that $\beta=0.5, \alpha=0.75, a=0.069, \mathrm{I}(\mathrm{u})=\frac{\mathrm{u}^{2}}{50}, \mathrm{~T}=100$.

Let $F(t, u(t))=\frac{u^{2}}{6}$, then it easy to verify the assumptions $\left(H_{1}\right)-\left(H_{3}\right)$ hold with $b=\frac{1}{50}, d=\frac{1}{6}, a_{1}=$ $0.066, a_{2}=0.07$.

We choose $\mu_{0}=0.41$, a direct calculation shows that

$$
\frac{7 \mathrm{~B}(\alpha, \mathrm{T})}{3|\cos (\pi \alpha)| \Gamma^{2}(2-\alpha) \mathrm{T}}<\mathrm{a}_{1}=0.066 \leqslant \mathrm{a}_{2}=0.07<\frac{|\cos (\pi \alpha)|}{\Gamma^{2}(\alpha)(2-\alpha)} \mathrm{T}^{2(\alpha-1)}, \quad \mu_{0} b=0.0082<0.00825=\frac{\mathrm{a}_{1}}{8} \text {. }
$$

Then our results can be applied to the problem (3.10), which shows that the problem (3.10) possesses an unbounded sequence of solutions in $\mathrm{E}_{0}^{\alpha}$ for $\lambda \in[0.3,61], \mu \in[0,0.41]$.

\section{Acknowledgment}

The authors thank the referees for their careful reading of the manuscript and insightful comments, which helped to improve the quality of the paper. We would also like to acknowledge the valuable comments and suggestions from the editors, which vastly contribute to the perfection of the paper. This work is supported by National Natural Science Foundation of China (No.11001274, 11101126, 11261010, 11301153, 61070247), China Postdoctoral Science Foundation (No.20110491249), Key Program of Henan Province (132102210246), Key Technologies R \& D Program of Education Department of Henan Province (13B510001), Key Scientific and Technological Research Project of Department of Education of Henan Province (NO. 12B110006,14A510015), Youth Science Foundation of Henan University of Science and Technology (NO. 2012QN010), and The Natural Science Foundation to cultivating innovation ability of Henan University of Science and Technology (NO. 2013ZCX020).

\section{References}

[1] C.-Z. Bai, Existence of three solutions for a nonlinear fractional boundary value problem via a critical points theorem, Abstr. Appl. Anal., 2012 (2012), 13 pages. 1

[2] G. Bonanno, S. A. Marano, On the structure of the critical set of non-differentiable functions with a weak compactness condition, Appl. Anal., 89 (2010), 1-10. 1 
[3] G. Bonanno, R. Rodríguez-López, S. Tersian, Existence of solutions to boundary value problem for impulsive fractional differential equations, Fract. Calc. Appl. Anal., 17 (2014), 717-744. 1, 1, 2, 2, 2

[4] J. Chen, X.-H. Tang, Existence and multiplicity of solutions for some fractional boundary value problem via critical point theory, Abstr. Appl. Anal., 2012 (2012), 21 pages. 1

[5] J.-N. Corvellec, V. V. Motreanu, C. Saccon, Doubly resonant semilinear elliptic problems via nonsmooth critical point theory, J. Differential Equations, 248 (2010), 2064-2091. 1

[6] A. M. A. El-Sayed, Nonlinear functional-differential equations of arbitrary orders, Nonlinear Anal., 33 (1998), 181-186. 1

[7] V. J. Erwin, J. P. Roop, Variational formulation for the stationary fractional advection dispersion equation, Numer. Methods Partial Differential Equations, 22 (2006), 558-576. 1

[8] B. Ge, Multiple solutions for a class of fractional boundary value problems, Abstr. Appl. Anal., 2012 (2012), 16 pages. 1

[9] Z.-G. Hu, W.-B. Liu, J.-Y. Liu, Ground state solutions for a class of fractional differential equations with Dirichlet boundary value condition, Abstr. Appl. Anal., 2014 (2014), 7 pages. 1

[10] F. Jiao, Y. Zhou, Existence of solutions for a class of fractional boundary value problems via critical point theory, Comput. Math. Appl., 62 (2011), 1181-1199. 1, 2.4, 2, 2, 2, 3

[11] F. Jiao, Y. Zhou, Existence results for fractional boundary value problem via critical point theory, Internat. J. Bifur. Chaos Appl. Sci. Engrg., 22 (2012), 17 pages. 1

[12] A. A. Kilbas, M. H. Srivastava, J. J. Trujillo, Theory and applications of fractional differential equations, North-Holland Mathematics Studies, Elsevier Science B.V., Amsterdam, (2006). 1, 2.1, 2.2, 2.3

[13] A. A. Kilbas, J. J. Trujillo, Differential equations of fractional order: methods, results and problems, I, Appl. Anal., 78 (2001), 153-192.

[14] V. Lakshmikantham, S. Leela, J. V. Devi, Theory of fractional dynamic systems, Camb. Sci. Publ., Cambridge, (2009). 1

[15] Y.-N. Li, H.-R. Sun, Q.-G. Zhang, Existence of solutions to fractional boundary-value problems with a parameter, Electron. J. Differential Equations, 2013 (2013), 12 pages. 1

[16] J. Mawhin, M. Willem, Critical point theory and Hamiltonian systems, Applied Mathematical Sciences, SpringerVerlag, New York, (1989). 1

[17] G. M. Mophou, Existence and uniqueness of mild solutions to impulsive fractional differential equations, Nonlinear Anal., 72 (2010), 1604-1615. 1

[18] N. Nyamoradi, R. Rodríguez-López, On boundary value problems for impulsive fractional differential equations, Appl. Math. Comput., 271 (2015), 874-892. 1, 1

[19] P. H. Rabinowitz, Minimax methods in critical point theory with applications to differential equations, CBMS Regional Conference Series in Mathematics, Published for the Conference Board of the Mathematical Sciences, Washington, DC; by the American Mathematical Society, Providence, RI, (1986). 1, 3

[20] R. Rodríguez-López, S. Tersian, Critical Point Theory and Hamiltonian Systems, Fract. Calc. Appl. Anal., 17 (2014), 1016-1038. 1, 1

[21] A. M. Samoǐlenko, N. A. Perestyuk, Impulsive differential equations, With a preface by Yu. A. Mitropolskiĭand a supplement by S. I. Trofimchuk, Translated from the Russian by Y. Chapovsky, World Scientific Series on Nonlinear Science, Series A: Monographs and Treatises, World Scientific Publishing Co., Inc., River Edge, NJ, (1995). 1

[22] H.-R. Sun, Q.-G. Zhang, Existence of solutions for a fractional boundary value problem via the Mountain Pass method and an iterative technique, Comput. Math. Appl., 64 (2012), 3436-3443. 1

[23] C. Torres, Mountain pass solution for a fractional boundary value problem, J. Fract. Calc. Appl., 5 (2014), 1-10.

[24] Y.-L. Zhao, H.-B. Chen, B. Qin, Multiple solutions for a coupled system of nonlinear fractional differential equations via variational methods, Appl. Math. Comput., 257 (2015), 417-427. 1 